\title{
Physico-chemical interactions between compartment- forming lipids and other prebiotically relevant biomolecules
}

\author{
F. Olasagasti ${ }^{1}$, M.-C. Maurel ${ }^{2}$, and D.W. Deamer ${ }^{3}$ \\ ${ }^{1}$ Department of Biochemistry and Molecular Biology, School of Pharmacy, University of the \\ Basque Country (UPV/EHU), 01006 Vitoria-Gasteiz, Basque Country, Spain \\ ${ }^{2}$ ANBioPhy, Molecular and Structural Archeobiology, UPMC Univ Paris 6, 75005 Paris, France \\ ${ }^{3}$ Dept. Biomolecular Engineering, University of California, Santa Cruz CA 95064, USA
}

\begin{abstract}
Lipids are essential constituents of contemporary living cells, serving as structural molecules that are necessary to form membranous compartments. Amphiphilic lipid-like molecules may also have contributed to prebiotic chemical evolution by promoting the synthesis, aggregation and cooperative encapsulation of other biomolecules. The resulting compartments would allow systems of molecules to be maintained that represent microscopic experiments in a natural version of combinatorial chemistry. Here we address these possibilities and describe recent results related to interactions between amphiphiles and other biomolecules during early evolution toward the first living cells.
\end{abstract}

\section{Introduction}

Compartmentalization of chemical systems was a necessary step in the formation of the first living cells (protocells) during prebiotic chemical evolution. In his seminal 1936 work on the origin of life, A.I. Oparin proposed that the first compartments might have been aggregated polymers he called coacervates [1]. This concept inspired early studies by Fox and co-workers who found that if amino acid mixtures were heated to the point that they became molten, a variety of chemical bonds were produced. The resulting polymers were referred to as proteinoids, and it was shown that under certain conditions the proteinoids assembled into microscopic spheres with diameters in the micrometer range [2]. A version of microspheres was recently investigated by Koga et al. [3] who created microscopic spherical compartments composed of nucleotides and a range of cationic oligopeptides or polypeptides. Unlike lipid vesicles, the microdroplets had no surface boundary structure, and their size and sedimentation properties depended on their composition.

Although such self-assembled structures are worthy of study, most research on protocells now focuses on molecular systems bounded by membranes. Such systems are becoming increasingly complex and the diverse nature of the component chemicals lends itself to a systems chemistry approach. Here we review physicochemical interactions between compartment-forming amphiphiles and other biomolecules that are relevant to the self-assembly of protocells in the prebiotic environment. 


\section{Interactions between nucleotides, nucleic acids and amphiphiles}

Amphiphiles are defined as compounds having both hydrophilic and hydrophobic groups on the same molecule. Hydrophobicity is imparted by non-polar hydrocarbon moieties, and hydrophilicity by polar or ionic groups, the most common being hydroxyl, carboxyl and phosphate. All lipids are amphiphilic, so here we will use the term lipid to denote biological amphiphiles such as fatty acids, phospholipids and sterols, all of which can be components of the lipid bilayers of biological membranes. We will use the more general term amphiphile for those lipid-like compounds that can be synthesized abiotically, for instance the products of Fischer-Tropsch reactions or those occurring in carbonaceous meteorites.

It is possible that amphiphilic compounds coexisted with amino acids and nucleotides during prebiotic chemical evolution. Powner et al. [4] examined the colocalization of nucleotides and amphiphiles that would result from the conditions required for synthesis. In particular, they investigated the phosphorylation of mixed length alkanols as a possible pathway for the formation of phosphorous-containing amphiphiles. These molecules would be comparable to the ubiquitous phospholipids in contemporary cells, and Powner et al. chose to study their synthesis under conditions that could have also promoted the formation of pyrimidine ribonucleotides by using phosphate as a general acid-base catalyst and buffer. Therefore, a key element in their proposed reaction system is phosphate, which could have come from schreibersite, a mineral present in ironnickel meteorites. Considering the geochemistry that is associated with iron-nickel meteorite impacts, it was suggested that primitive oceans would evaporate following an event similar to the giant impact that formed Mare Imbrium on the moon. During evaporation of early oceans at high pressure and temperatures, the Kölbel-Engelhard variant of the Fischer-Tropsch reaction would have incorporated $\mathrm{CO}$ produced when $\mathrm{CO}_{2}$ was reduced by Ni-Fe particles in the atmosphere. The KölbelEngelhard reaction leads to more oxygen-containing species than the Fischer-Tropsch reaction, so that straight chain alkanols, ketones, aldehydes (including $\mathrm{H}_{2} \mathrm{CO}$ ) and carboxylic acids are formed in addition to straight chain hydrocarbons. Powner et al. reacted a representative mixture of alcohols (hexanol, decanol and a large fraction of ethanol) with ammonium phosphate and urea by drying and heating them on a glass fiber support to simulate spreading on an inert surface. They detected decyl phosphate and hexyl phosphate at 7:3 ratios but almost no ethyl phosphate. The authors attributed this selectivity to the reversibility of the phosphorylation reaction and the lower volatility of the reagents that produced the main products. For instance, the volatility of decanol is lower than hexanol, which in turn is lower than ethanol. During prebiotic chemical evolution, the longer chain alkyl phosphates could have mixed with alkyl alcohols, resulting in vesicles with novel properties like those discussed in section 5 below.

If lipids and nucleotides coexisted during the early stages of chemical evolution, it is interesting to study possible interactions of these two kinds of molecules, with particular attention to the formation of the first nucleic acids. This question will be addressed in the context of a plausible prebiotic scenario in which small volumes of organic solutes could have undergone anhydrous and hydrated fluctuations in intertidal zones of volcanic land masses or in lacustrine environments produced by precipitation $[5,6]$. The fluctuating conditions would serve several purposes. First, the concentrating effect occurring during evaporation would produce films of reactive organic solutes on dry mineral surfaces. Second, the anhydrous state provides a chemical potential that can drive condensation reactions essential for polymer synthesis. Finally, upon rehydration any amphiphilic molecules that are present will assemble into membranous vesicles that encapsulate polymeric products. Most would be inert, but a rare few presumably happened to contain systems of polymers which could catalyze a primitive metabolism as well as further growth by polymerization. This mixture of inert and active compartments represents a natural version of combinatorial chemistry which had the potential to undergo Darwinian selection as active compartments grew at the expense of inert compartments. Here we will refer to the active compartments as protocells. 


\subsection{Elongation by guided polymerization}

When amphiphilic molecules in an aqueous environment are concentrated by evaporation, they form a multilamellar liquid-crystalline structure in which the solutes can be trapped between the lipid layers [7]. In this environment, monomers that freely diffuse in a dilute bulk phase are concentrated within a two dimensional space, but are still able to diffuse between the lipid layers and react to form a condensation product. The dry phase of the dry/wet cycles that would happen during low tides favors condensation reactions because chemical activity of water would be extremely low between the lipid layers, favoring reactions in which water becomes a leaving group and ester bonds form. In order to test this hypothesis, Rajamani et al. [5] heated dry mixtures of lipids and ribonucleotide monophosphates under different dry atmospheres and tested different lipids, ribonucleotide to lipid ratios, heating temperatures, reaction times and number of dry/wet reaction cycles. These experiments showed that long polymers corresponding to 20 to 100 nucleotides in length were synthesized. Mixtures of 1-palmitoyl-2-oleoyl-SN-glycero-3-phosphatidic acid (POPA) and AMP at 1:8 lipid:monomer ratios provided the highest product yields when the mixture was heated for 2 hour intervals during 5 dry/wet cycles.

\subsection{Potential for replication}

It is interesting to note that the organizing effect of lipids is not limited to the monomers of biomolecules that could have constituted the first living cells. They could also organize macromolecules in these environments and promote the emergence of important biological processes such as the transfer of information. Olasagasti et al. [6] tested this hypothesis using the aforementioned system, with the addition of a 64-mer deoxyoligonucleotide. The reaction mixture was dried and heated as described above, resulting in a measurable amount of product that was presumably double stranded. All these reactions were carried out with non-activated mononucleotides and reaction yields were low. However, the authors excluded the possibility of random synthesis by a comprehensive set of controls, by sequencing the reaction products, and by comparing their sequences with random sequences. They concluded that the match between reaction products and the template represented transfer of sequence information promoted by the organizing effect of the lipid environment.

The lipids were still capable of forming vesicles after several reaction cycles, and the vesicles would readily encapsulate reaction products. The co-localization of a replicating molecular system within a membranous boundary would lead directly to the emergence of protocells, whereas other scenarios that are considered plausible for the synthesis of nucleic acids such as mineral surfaces [810], would require additional steps in which polymers are first released from the mineral, followed by encapsulation.

\section{Interactions with amino acids}

Interactions between amphiphilic compounds and amino acids have also been investigated. For instance, Imai et al. [11] fabricated a simulated hydrothermal vent in which circulating sea water at high pressure and temperature was quenched by passage into low pressure and temperature conditions. When $100 \mathrm{mM}$ glycine was included, glycine dimers and trimers were detected, suggesting that peptide bonds were thermodynamically favored at high temperature, then preserved by quenching. Significantly, it was observed that including oleic acid, a self-assembling amphiphile, markedly enhanced the yield by 15 -fold [12].

Zepik et al. [13] incubated vesicles assembled from didodecyldimethylammonium bromide (DDAB) with thioglutamic acid (an activated form of glutamic acid) for times ranging from zero to several days in order to study the formation of oligomers of glutamic acid. DDAB vesicles have a permanent positive charge on the quaternary ammonium of each molecule, and the expectation was that the electrostatic attraction between negatively charged glutamic acid and positively charged vesicle surfaces could concentrate glutamic acid monomers and promote their polymerization. After 4-5 days of incubation at $0^{\circ} \mathrm{C}$, Zepik et al. were able to detect oligomers as long as 11 mers by using 
HPLC and MALDI-TOF-MS. In addition, the presence of glutamic acid oligomers promoted the aggregation of DDAB vesicles, confirming the interaction between both kinds of molecules. In the absence of lipids, no oligomer appeared longer than tetramers. There was a clear temperature dependence for the oligomerization reaction, with oligomer formation inhibited at higher temperatures. Zepik et al. interpreted these findings as a reaction mechanism governed by the adsorption of dimers to the lipid surface. This process could promote the concentration and further polymerization of the monomers, but it would be less efficient at higher temperatures.

This study also investigated elongation of glutamic acid oligomers encapsulated in dimyristoyl phosphatidylcholine (DMPC) vesicles, with thioglutamic acid delivered from outside of the vesicles as a kind of feedstock. The passive diffusion of the activated monomers through the DMPC membrane was sufficient to support elongation of the internal oligoglutamate by a non-enzymatic polymerization reaction.

\section{General lipid-macromolecule interactions}

Some compartment-related phenomena involve interactions between lipids and other molecules. For instance, Pereira de Souza et al. [14] prepared 1-palmitoyl-2-oleoyl-sn-glycero-3phosphatidylcholine (POPC) vesicles in the presence of different ribosome concentrations, as well as a complete transcription/translation system. As the ribosome concentration increased, it was expected that a Poisson distribution would produce some vesicles with no ribosomes, some with a single ribosome, some with two, but few with more than two. However, they unexpectedly found that empty liposomes coexisted with liposomes that had encapsulated very large numbers of ribosomes. When they tested the encapsulation of the complete transcription/translation machinery, their findings were also consistent with a non-Poissonian distribution of vesicle content, such that the distribution of vesicle contents followed a power-law distribution in both cases.

From these results the authors deduced that cooperative interactions take place during the encapsulation of solutes at the time when vesicles are formed. These researchers suggested that the mechanism for this vesicle crowding effect is related to weak and short-lived solute-solute and solute-membrane interactions. Ribosome binding would be unlikely to account for the observed phenomenon by itself because of the low concentrations used for the experiments. Instead, a more plausible explanation is that the presence of empty and solute-filled vesicles was the stochastic outcome of competition between vesicle closure and solute capture rates. In the absence of solutes, flat lipid bilayers would rapidly form empty vesicles. However, their closure rate would be slower if solutes were non-specifically adsorbed to the bilayer, so that more solutes could accumulate on the membrane and thereby produce some crowded vesicles and some empty ones.

Van Hoof et al. [15] carried out a related study that provides more insight into the crowding of encapsulated solutes during the formation of vesicles. As noted by Pereira de Souza et al., it is difficult to experimentally analyze short-lived intermediates during vesicle formation. Therefore, Van Hoof et al. used molecular dynamics simulations to study the spontaneous transition from flat bilayers to vesicles in the absence and presence of solvated proteins and with different proteinmembrane interactions. These authors observed that the membrane bulges out during the bilayervesicle transition. This bulging is accompanied by an influx of solvent, which depends on the rate of diffusion. It follows from these results that if there was no additional force to drive the proteins inward, such as a stronger protein-membrane interaction, the proteins (with a larger size, and therefore, slower diffusion than water) would have a lower concentration inside the vesicles. These researchers suggested that membrane components and $\mathrm{pH}$ could be adjusted for the membrane surface to strongly attract the elements of the RNA replication system used by Pereira de Souza et al. In this way, the concentration of solutes inside the vesicles could be predictably enhanced.

The interactions of lipid vesicles with the solutes inside and outside of the vesicles may contribute, among other phenomena, to the budding and division of the vesicles themselves. Budin et al. [16], Hanczyc et al. [17] and Terasawa et al. [18] among others have addressed possible ways for primitive vesicles to bud and divide. In particular, Terasawa et al. showed how biopolymers could 
favor the growth and division of vesicles by budding, which is a process comparable to the division mechanism in L-form bacteria[19]. Terasawa et al. created giant unilamellar vesicles by electroformation, which favored the fusion of smaller vesicles. After fusion, they stopped the electric potential and observed that when polyethyleneglycol 6000 polymer (PEG 6000) was encapsulated, the vesicles budded and elongated, resulting in torus, horseshoe, and elongated tube shapes. In the absence of PEG the vesicles remained mostly spherical with multivesicular structures produced by invagination, whereas in the presence of PEG, the extent of membrane invagination was reduced. The authors explained these phenomena as the depletion of PEG on the membrane surface, a property that can also be used to induce vesicle fusion and that could cause vesicle budding under certain conditions. It is particularly noteworthy that vesicle budding did not occur when PEG was present in both the external and internal solutions of the vesicles. The model based on the depletion effect was consistent with several observations, including a more rapid budding transformation at higher concentrations and/or higher polymer molecular weights. This finding can be explained by the fact that with a higher free energy difference between the bulk and depletion volume, the membrane still maintains its local curvature during fusion, resulting in rapid budding after fusion is completed.

In a related study, Kurihara et al. [20] made giant vesicles by mixing POPC, palmitoyl oleoyl phosphatidylglycerol (POPG), 1-octadecyl imidazole and an additional positively charged molecule they called V (N, N-dimethyl-N-[12((4'-formyl) phenoxy) dodecyl]-N-dodecyl-ammonium bromide). They encapsulated polymerase chain reaction (PCR) solutions inside the vesicles and observed that the addition of a $\mathrm{V}$ molecule precursor during amplification resulted in vesicle budding and division, which was more pronounced with higher DNA concentrations. To explain their results, they suggested that when DNA is synthesized in the vesicle, it binds and concentrates the V molecule on the inner leaflet of the membrane, which creates an imbalance with respect to the outer leaflet. DNA can also be trapped inside the lipid membrane, thereby forming a lipoplex. When the cationic precursor of $\mathrm{V}$ is added to the external solution, the entrapped DNA increases the likelihood that the precursor will be turned into the final membrane component by a membrane-bound catalyst (1-octadecyl imidazole). As a result, membrane component $\mathrm{V}$ remains confined to the area of the vesicle where the DNA is bound, eventually leading to vesicle budding and division. Since DNA promotes the accumulation of the precursor, higher concentrations of DNA result in preferential budding and division of the giant vesicles. This system has a limitation in that the composition of the membrane molecules gradually changes during the reaction so that percentage of phospholipids decreases as the percentage of cationic membrane molecules increases. However, it is important to note that this system connects the mechanism of vesicle division with DNA amplification, a feedback process that would be necessary for the functioning of protocells.

\section{Interactions between aliphatic amphiphiles}

In addition to the interactions between lipids and solutes, lipid-lipid interactions can also result in properties that could have provided an evolutionary advantage for primitive protocellular systems. Namani et al. [21] prepared one such system by mixing fatty acids and alkyl amines, and demonstrated that vesicles formed from such mixtures are surprisingly stable at high and low $\mathrm{pH}$ ranges. This stability depends on the potential for hydrogen bonding between head groups of the component amphiphiles, and also on the net charge of the resulting lipid bilayer. At high $\mathrm{pH}$ values, the fatty acids are negatively charged and form hydrogen bonds with the neutral amine head groups. At low $\mathrm{pH}$ values, the amine groups are positively charged and form hydrogen bonds with the neutral carboxyl groups of the fatty acids. In addition to making observations at extreme $\mathrm{pH}$ values, this work also tested the stability of these mixed vesicles in the presence divalent ions that may have been present in primitive oceans. The alkylamine/fatty acid vesicles in low $\mathrm{pH}$ ranges are stable at $0.1 \mathrm{M}$ concentrations of $\mathrm{MgCl}_{2}$ or $\mathrm{CaCl}_{2}$, but at alkaline $\mathrm{pH}$ values, even low concentrations of divalent cations $\left(<0.1 \mathrm{mM} \mathrm{Mg}^{+2}\right)$ affected the stability of vesicles. The authors explained these results by the fact that the neutral (protonated) fatty acids and positively charged alkyl ammonium molecules do not undergo electrostatic association with cations at low $\mathrm{pH}$, whereas at high $\mathrm{pH}$ the 
negative charges of the fatty acids bind divalent cations, thereby causing aggregation to form calcium or magnesium soaps. This hypothesis was studied more thoroughly by Caschera et al. [22] who analyzed monolayers composed of fatty acids, fatty dioic acids, and alkyl amines. They concluded that the stabilization can be attributed to the formation of hydrogen bonds between protonated and deprotonated acids and the electrostatic interactions between the ammonium and carboxylate head groups.

Rendon et al. [23] tested the stability and encapsulation properties of vesicles formed from oleic acid when alkyl alcohols or alkanes were added. By taking the cac (critical aggregation concentration) as a measure of the stability of these mixed systems, they found that the addition of alkanes to oleic acid vesicles did not affect the cac value regardless of the chain length of the alkanes in the mixture. However, when they used alkyl alcohols, they found that alcohols with alkyl chains in the 10- to 14-carbon range produced larger vesicles. This effect was more pronounced when the alkyl alcohol to oleic acid ratio was 1:2. When they used alkanes mixed with oleic acid, the turbidity of the solutions that contained the mixture remained similar to pure oleic acid/oleate samples within the range of alkane to oleic acid ratios that they tested (1:10 to $1: 2)$.

These authors also studied the capability of mixed vesicles to encapsulate and retain solutes. In this regard, two processes were analyzed: the initial release rate of entrapped dyes in mixtures of oleic acid with alkanes or alkyl alcohols and the final amount of entrapped dye after apparent equilibrium was reached. Addition of the alkanes and alkyl alcohols having varying chain lengths reduced the initial dye release rate, but the amount of remaining encapsulated dye was lower when alkanes and alkyl alcohols with shorter chains were in the mix. This effect is related to a stabilization mechanism in which alkyl alcohols would have polar head interactions with the carboxylic groups of the oleic acid, while hydrophobic chain interactions would induce defects in the lipid bilayers when $\mathrm{C}-10$ to $\mathrm{C}-14$ chains were used.

\section{Lipid-PAH interactions}

Namani et al. [21] also investigated the effect of polycyclic aromatic hydrocarbons (PAHs) on membrane stability, and reported that small amounts of PAHs had a stabilizing effect on the bilayer permeability of akyl amine/fatty acid vesicles. It was suggested that PAH species could have played a role similar to that of cholesterol in contemporary membranes. Groen et al. [24] tested this idea with PAH species such as pyrene, 1-hydroxypyrene, 9-anthracene carboxylic acid, 1-pyrene carboxaldehyde and 9-fluorenone. They found that the PAHs could be incorporated into decanoic acid vesicles at different fatty acid:PAH ratios ranging from 200:1 to 20:1. These authors confirmed the earlier report of Namani et al. that PAH species could stabilize fatty acid vesicles, and provided quantitative information to support this effect. Specifically, incorporation of 1:10 mole ratios of 1hydroxypyrene/ decanoic acid lowered the initial rate of $\mathrm{KCl}$ permeation by 4.2 fold while 1:10 9anthracene carboxylic acid/decanoic acid lowered the permeation of $\mathrm{KCl}$ by 2.5 fold. It is significant that this permeability reduction is the same order of magnitude as the effect of cholesterol in decreasing $\mathrm{K}^{+}$and $\mathrm{Na}^{+}$permeation of phospholipid membranes. Groen et al. also measured PAH effects on permeability to sucrose for decanoic acid vesicles, observing that 1-hydroxypyrenecontaining vesicles showed the most marked reduction in permeability to sucrose (from $1.3 \times 10^{-8}$ $\mathrm{cm} / \mathrm{s}$ to $3.3 \times 10^{-9} \mathrm{~cm} / \mathrm{s}$ ). A reasonable conclusion is that mixtures of different fatty acids, functional alkyl molecules, and PAHs in the prebiotic environment may have created a variety of vesicles with a range of permeabilities for different molecules. Some vesicles in this range would have permeability properties that would allow entry of nutrient solutes, yet prevent loss of polymeric products, thus allowing accumulation inside the vesicles with an ensuing evolution towards increasingly functional encapsulated systems of polymers.

In addition to controlling the fluidity and the permeability of membranes, PAHs have been proposed as possible primitive membrane-bound energy transducers. Electron transport, proton pumping and carbon dioxide fixation are essential to all life today, and several PAH derivatives have pigment properties that allow them to take part in relevant photochemical reactions [25]. For 
instance, pyrene when present in liposome membranes can enter an excited state upon illumination and donate electrons to benzophenone [26]. Furthermore, both 1-hydroxy naphthol and 9-hydroxy 1, 4,5 sulfopyrene change their pKa by orders of magnitude upon illumination and release a proton [27]. When illuminated in a non-polar solvent, phenanthrene is able to fix carbon dioxide as phenanthrene carboxylic acid [28].

A recent test of PAH as a transducer of electron transport was carried out by Cape et al. [29] who used a simple photochemical trans-membrane charge transfer reaction in which $\mathrm{Fe}(\mathrm{CN})_{6}^{-{ }^{-3}}$ could be reduced to $\mathrm{Fe}(\mathrm{CN})_{6}^{-4}$ inside decanoic acid vesicles while EDTA (ethylenediaminetetraacetic acid) was oxidized outside. They used naphtho[2,3a]-pyrene and perylene as PAH catalysts for the photochemical reaction. These authors observed that the structural integrity of the vesicles was maintained throughout the photochemical redox reaction. They also found that EDTA did not quench the fluorescence of the PAHs in the membrane, that the overall rates of reaction exhibited non-linear sensitivity to the PAHs and to the encapsulated $\mathrm{Fe}(\mathrm{CN})_{6}^{-3}$, and that the estimated driving force for oxidative quenching by $\mathrm{Fe}(\mathrm{CN})_{6}{ }^{-3}$ was greater than the reductive quenching by EDTA with either of the PAHs that they used. Cape et al. proposed that the reaction mechanism involved the oxidation of membrane bound PAHs by $\mathrm{Fe}(\mathrm{CN})_{6}^{-3}$ (forming a PAH radical), followed by the oxidation of EDTA on the other side of the membrane.

It is important to note that the fatty acid vesicles must allow fast diffusion of ions through the membrane in order for electroneutrality to be preserved during charge transport. Therefore, it seems that fatty acid vesicles that included PAHs could have provided suitable compartments for the encapsulation of primitive chemical systems. Despite the ongoing progress in this area, there are additional considerations about the feasibility of systems such as that presented by Cape et al. It is not clear how the trans-membrane redox potential could be coupled to drive metabolic reactions that would provide an evolutionary advantage for emerging protocells. In addition, although they suggest that photooxidative activation of small molecules such as alcohols could be one way to direct photochemical energy into chemical bond energy, most organic reactions require two-electron chemistries. Some sort of conversion would be required in order to derive two-electron chemistry from a one-electron photochemical model system.

\section{Compartments with more complex amphiphiles}

The fatty acid esters of glycerol can also increase the stability of lipid vesicles. Maurer et al. [30] tested mixtures of 8-, 10-, 12- and 18-carbon fatty acids and their corresponding glycerol esters in terms of the concentrations required for vesicle formation. Glycerol monoacyl amphiphile derivatives (GMAs) decreased the concentration of amphiphiles between 4- to 9-fold, depending on the length of the carbon chain. GMAs were more effective at reducing the critical vesicular concentration ( $\mathrm{cvc}$ ) of shorter chain length fatty acids. They proposed that polar interactions between head groups may be primary factors in stabilizing shorter chain vesicles, while longer chain vesicles are stabilized by hydrophobic interactions between the tails.

In addition to using cve as a measure of stability, these authors studied the ability of GMA/fatty acid vesicles to encapsulate carboxyfluorescein ( $\mathrm{CF}$, molecular weight $376.7 \mathrm{Da}$ ) and tRNA (molecular weight $\sim 25 \mathrm{kDa}$ ). Although an 8 carbon fatty acid (octanoic acid) can assemble into vesicles at sufficiently high concentrations, the bilayers are highly dynamic and cannot maintain gradients of CF or tRNA for any length of time even if the corresponding GMA is added. Decanoic acid vesicles were also unable to encapsulate these solutes, but when decanoic acid monoglyceride was added to the mix tRNA could be encapsulated for at least one hour, but not CF. The results obtained for lauric acid were comparable to the decanoic acid/glycerol monodecanoate system and from this chain length on, all systems were able to encapsulate both molecules (CF and tRNA) for at least one hour. These authors observed that lauric acid vesicles were unstable at $25^{\circ} \mathrm{C}$, near the Tm of this fatty acid, so the experiments were carried out at $32^{\circ} \mathrm{C}$, at which point they were stable. On the other hand, if vesicles with lauric acid and glycerol monolaureate were formed at $32^{\circ} \mathrm{C}$, they remained stable at $20^{\circ} \mathrm{C}$ for weeks. 
This investigation showed that the formation of the fatty acid ester of glycerol would have been an important enhancement of fatty acid membrane stability. It may be significant that most membrane phospholipids now incorporate glycerol as a backbone, so the glycerol ester bond could have been one of the first evolutionary steps toward the lipid composition of today's cell membranes.

We can now ask whether it is plausible that monoglycerides were available in the prebiotic environment. This question was addressed by Simoneit et al. [31], who tested whether acylglycerols could be synthesized under simulated hydrothermal conditions. These authors heated an aqueous mixture of n-alkanoic acids with glycerol at $150^{\circ} \mathrm{C}$ for $72 \mathrm{~h}$. Furthermore, they also tested the same mixtures with an addition of oxalic acid, which are subject to a disproportionation reaction above $150^{\circ} \mathrm{C}$ that yields formic acid and $\mathrm{CO}_{2}$. Formic acid in turn decomposes to form $\mathrm{CO}_{2}, \mathrm{CO}, \mathrm{H}_{2}$ and $\mathrm{H}_{2} \mathrm{O}$. As a result, the addition of oxalic acid provides reducing power in the form of hydrogen.

Under these conditions, Simoneit et al. reported a total reaction yield of $42.1 \pm 7.4 \%$, in which the major products were monoacylglycerols, followed in abundance by diacylglycerols, triacylglycerols, and to a less extent, methyl alkanoates. The authors found glyceryl mono- and dialkanoate esters of n-alkanoic acids composed of 7- to 16-carbon chains. Glycerol trialkanoate esters were more rare and they did not find any detectable amount for n-alkanoic acids of 12-, 14-, 15- or 16-carbon chains. From these results, it is apparent that reaction conditions that simulate the physicochemical environment of volcanic hydrothermal sites can produce fatty acid esters of glycerol. Simoneit et al. also showed that these products could self-assemble to form membranous structures, such as multilamellar globular structures with smaller membranous vesicles budding from the surface as long as the environmental conditions allowed for the fluid state of the membranes. In this regard, it is worth mentioning that while $\mathrm{C}_{10}$ and $\mathrm{C}_{12}$ monoglycerides did present vesicles that were budding from the multilamellar structures, no vesicle was detected from the multilamellar structures formed from monoglycerides with longer carbon chains.

Relatively high temperatures and incubation times in sealed containers were used by Simoneit et al. to synthesize monoglycerides, and this might be a concern in terms of plausibility. However, it is possible for glycerol esters to form at lower temperatures and atmospheric pressure [32]. If an aqueous solution of glycerol and decanoic acid is maintained at temperatures between $50-90^{\circ} \mathrm{C}$, surprisingly high yields of glycerol monodecanoate are produced in a few hours even in the presence of water. The reaction can occur because decanoic acid is immiscible in water, so that polar and nonpolar phases are present. The esterification reaction presumably occurs at the interface and the product dissolves in the non-polar phase, thereby preventing the back reaction of hydrolysis.

From the investigations described so far, it seems likely that compartments formed with mixed amphiphiles could have resulted in evolutionary advantages among the first protocells. This idea was advanced by Cheng et al. [33], Chen et al. [34], and more recently by Budin et al. [16], who studied the effect of adding phospholipids to fatty acid vesicles. Budin et al. used oleate fatty acid vesicles that contained $10 \mathrm{~mol} \%$ di-oleoyl-phosphatidic acid (DOPA). When they mixed these vesicles with 1 equivalent of pure oleate vesicles, they observed an increase of about $16 \%$ in the size of vesicles by using Förster resonance energy transfer (FRET). The growth of the vesicles involved a filamentous shape transition and eventually the vesicles divided. The authors proposed that the increased size of vesicles with phospholipids could be explained by the fact that fatty acids were continuously entering and leaving the membrane from the bulk aqueous phase, a process with characteristic off rates and on rates. If phospholipid reduced the rate at which fatty acids left the membrane, the result would be a growth of the vesicle because of fatty acid accumulation. They suggested that this effect is stronger with phospholipids whose head groups form stronger hydrogen bonds with the oleate molecules. They also observed that phospholipids with saturated fatty acids, which produced stronger interacyl chain van der Waals interactions, formed more ordered membranes which reduced the off-rate of fatty acids more than phospholipids with unsaturated chains.

The authors concluded that lower membrane fluidity is one of the effects that drives the growth of the phospholipid vesicles at the expense of the pure fatty acid vesicles which decreased in size. However, they also observed that the lower fluidity of the phospholipid-containing vesicles would reduce permeability to nutrient monomers required for biopolymer synthesis. Other solutions would 
be required for uptake of nutrients, for instance, insertion of peptides into the membranes that could serve as transporters or channels.

Monnard et al. [35] addressed the problem of low membrane permeability to polar ionic solutes by investigating a model system of dimyristoylphosphatidylcholine (DMPC) liposomes with an encapsulated T7 RNA polymerase that is optimally active at $37^{\circ} \mathrm{C}$. DMPC has a Tm of $23^{\circ} \mathrm{C}$, a temperature at which the bilayer is in the fluid state with gel phase islands still present. The islands produce ion conducting defects around their periphery [36] which cause permeability to be two orders of magnitude higher than at temperatures above or below the Tm. Monnard et al. made use of this property by cycling the incubation temperature between $23.3^{\circ} \mathrm{C}$ and $37^{\circ} \mathrm{C}$ with a PCR device. The question was whether the higher permeability at $23^{\circ} \mathrm{C}$ was sufficient to allow diffusion of the substrate nucleotide triphosphates into the liposomes and supply the polymerase. The system was cycled through a period of high NTP diffusion with low T7 RNA polymerase activity, followed by a period of low NTP diffusion with high T7 RNA polymerase activity.

The authors found that the temperature cycling allowed the concentration of NTPs to be sufficiently high inside the vesicles for the T7 RNA polymerase to synthesize RNA from a DNA template that was encapsulated with the enzyme. Although it is unlikely that protocells had phospholipid membranes, given that cycling conditions could have served as an energy source for the synthesis of biomolecules [5, 6, 13], it seems reasonable that both temperature cycles and anhydrous-hydration cycles would be part of the process leading to the emergence of protocells. As evolutionary selection began in protocell populations, the cycling would give way to more sophisticated systems that included a primitive metabolism, biosynthesis of biopolymers, energy transduction through linked reactions and membrane transport mechanisms such as those investigated by Budin et al. [16].

\section{Conclusion}

From the evidence presented here, we propose that the role of amphiphilic molecules in prebiotic evolution could go beyond simple compartmentalization. Perhaps most important is the manner in which amphiphiles self-assemble into organized structures that can impose order and concentrate otherwise disordered solutes. It has long been considered that certain crystalline minerals such as clays and pyrite could have an organizing effect on organic compounds that adsorb to their surfaces. Here we described how liquid crystalline structures produced by self-assembly of amphiphilic compounds can also organize potential monomers and thereby promote the synthesis of polymers relevant to the function of protocells. Another important property of amphiphiles assembled as bilayer membranes is selective permeability to solutes, such that small molecules can enter a protocell but then accumulate in the form of less permeable polymers. This results in vast numbers of encapsulated polymers, each different from all the rest, and each a kind of experiment in a natural version of combinatorial chemistry. Finally, we note that bilayers are uniquely well suited to provide a hydrophobic site in which potential pigment molecules such as PAHs can be concentrated by partitioning. Different PAH species can undergo a variety of photochemical reactions that are relevant to using light as an energy source, including electron transfer, proton production and carbon dioxide fixation. This may provide a clue into how simple photochemistry could develop into a functional photosynthetic system. We hope that the points made in this review will convince others that the properties of amphiphiles are well worth exploring in relation to the origin of cellular life.

\section{References}

1. A. I. Oparin, The Origin of Life (Dover, New York, 1952)

2. S. W. Fox, K. Harada and J. Kendrick, Science 129, 1221-1223 (1959)

3. S. Koga, D. S. Williams, A. W. Perriman and S. Mann, Nat. Chem. 3, 720-724 (2011) 
4. M. W. Powner and J. D. Sutherland, Philos. Trans. R. Soc. B-Biol. Sci. 366, 2870-2877 (2011)

5. S. Rajamani, A. Vlassov, S. Benner, A. Coombs, F. Olasagasti and D. Deamer, Orig. Life Evol. Biosph. 38, 57-74 (2008)

6. F. Olasagasti, H. J. Kim, N. Pourmand and D. W. Deamer, Biochimie 93, 556-561 (2011)

7. D. Deamer, Chem. Soc. Rev. 41, 5375-5379 (2012)

8. P. C. Joshi, M. F. Aldersley, J. W. Delano and J. P. Ferris, J. Am. Chem. Soc. 131, 1336913374 (2009)

9. J. P. Ferris, A. R. Hill, R. H. Liu and L. E. Orgel, Nature 381, 59-61 (1996)

10. S. E. McGlynn, I. Kanik and M. J. Russell, Philos. Trans. R. Soc. A-Math. Phys. Eng. Sci. 370, 3007-3022 (2012)

11. E. Imai, H. Honda, K. Hatori, A. Brack and K. Matsuno, Science 283, 831-833 (1999)

12. H. Tsukahara, E. Imai, H. Honda, K. Hatori and K. Matsuno, Orig. Life Evol. Biosph. 32, 13$21(2002)$

13. H. H. Zepik, S. Rajamani, M. C. Maurel and D. Deamer, Orig. Life Evol. Biosph. 37, 495-505 (2007)

14. T. P. de Souza, F. Steiniger, P. Stano, A. Fahr and P. L. Luisi, ChemBioChem 12, 2325-2330 (2011)

15. B. van Hoof, A. J. Markvoort, R. A. van Santen and P. A. J. Hilbers, J. Phys. Chem. B 116, $12677-12683$ (2012)

16. I. Budin and J. W. Szostak, Proc. Natl. Acad. Sci. U. S. A. 108, 5249-5254 (2011)

17. M. M. Hanczyc, S. M. Fujikawa and J. W. Szostak, Science 302, 618-622 (2003)

18. H. Terasawa, K. Nishimura, H. Suzuki, T. Matsuura and T. Yomo, Proc. Natl. Acad. Sci. U. S. A. 109, 5942-5947 (2012)

19. J. Errington, Open Biol. 3, 120143 (2013)

20. K. Kurihara, M. Tamura, K. Shohda, T. Toyota, K. Suzuki and T. Sugawara, Nat. Chem. 3, 775-781 (2011)

21. T. Namani and D. W. Deamer, Orig. Life Evol. Biosph. 38, 329-341 (2008)

22. F. Caschera, J. B. de la Serna, P. M. G. Loffler, T. E. Rasmussen, M. M. Hanczyc, L. A. Bagatolli and P. A. Monnard, Langmuir 27, 14078-14090 (2011)

23. A. Rendon, D. G. Carton, J. Sot, M. Garcia-Pacios, R. Montes, M. Valle, J. L. R. Arrondo, F. M. Goni and K. Ruiz-Mirazo, Biophys. J. 102, 278-286 (2012)

24. J. Groen, D. W. Deamer, A. Kros and P. Ehrenfreund, Orig. Life Evol. Biosph. 42, 295-306 (2012)

25. D. W. Deamer, Adv. Space Res. 12, 183-189 (1992)

26. J. R. Escabiperez, A. Romero, S. Lukac and J. H. Fendler, J. Am. Chem. Soc. 101, 2231-2233 (1979)

27. R. Knochenmuss and S. Leutwyler, J. Chem. Phys. 91, 1268-1278 (1989)

28. M. Kanehara and K. Tsujimoto, Nippon Kagakkai Koen Yokoshu 79, 729 (2001)

29. J. L. Cape, P. A. Monnard and J. M. Boncella, Chem. Sci. 2, 661-671 (2011)

30. S. E. Maurer, D. W. Deamer, J. M. Boncella and P. A. Monnard, Astrobiology 9, 979-987 (2009)

31. B. R. T. Simoneit, A. I. Rushdi and D. W. Deamer, Adv. Space Res. 40, 1649-1656 (2007)

32. C. L. Apel and D. W. Deamer, Orig. Life Evol. Biosph. 35, 323-32 (2005)

33. Z. L. Cheng and P. L. Luisi, J. Phys. Chem. B 107, 10940-10945 (2003)

34. I. A. Chen, R. W. Roberts and J. W. Szostak, Science 305, 1474-1476 (2004)

35. P. A. Monnard, A. Luptak and D. W. Deamer, Philos. Trans. R. Soc. B-Biol. Sci. 362, 1741 1750 (2007)

36. S. G. Clerc and T. E. Thompson, Biophys. J. 68, 2333-2341 (1995) 